\title{
Impact of Blood Urea Nitrogen and Creatinine Levels on Maternal and Fetal Outcomes of Pregnancy: a Retrospective Cohort Study
}

\section{Guifang Deng ( $\square$ misyfly@163.com )}

Union Shenzhen Hosipital of Huazhong University of Science and Technology https://orcid.org/00000002-9520-2793

\section{Lanlan Wu}

Union Shenzhen Hospital of Huazhong University of Science and Technology

Yao Liu

Union ShenzhenHospital of Huazhong University of Science and Technology

Zengyou Liu

Union Shenzhen Hospital of Huazhong University of Science Technology

\section{Hengying Chen}

Shantou University Medical College

\section{Siwen Shen}

Union Shenzhen Hospital of Huazhong University of Science and Technology

\section{Yuanhuan Wei}

Union Shenzhen Hospital of Huazhong University of Science and Technology

\section{Ruifang Sun}

Union Shenzhen Hospital of Huazhong University of Science and Technology

\section{Research article}

Keywords: Urea nitrogen, Creatinine, Adverse pregnancy outcomes, Premature rupture of membranes, Macrosomia, Small for gestational age infants, Large for gestational age infants

Posted Date: April 1st, 2021

DOI: https://doi.org/10.21203/rs.3.rs-379457/v1

License: (1) (1) This work is licensed under a Creative Commons Attribution 4.0 International License. Read Full License 


\section{Abstract}

Background: Blood urea nitrogen (BUN) and creatinine (SCr) are associated with gestational diabetes mellitus (GDM). However, there were limited data in the literature on the influence of BUN and SCr on maternal and fetal outcomes of pregnancy. We aimed to examine the association of BUN and SCr levels during gestation with the risk of selected adverse pregnancy outcomes.

Methods: This retrospective cohort study included 1606 singleton mothers aged 22-44 years. Both BUN and SCr levels were collected and measured during the second (16-18 ${ }^{\text {th }}$ week), third (28-30 ${ }^{\text {th }}$ week) trimesters of gestation respectively and followed up pregnancy outcomes. Statistical analysis was used multivariate logistic regression.

Results: In the multivariate adjusted logistic regression model, the highest level of SCr in the second trimester increased the risk of PROM by $45 \%(95 \% \mathrm{Cl}, 1.01-2.09)$. In the third trimester of gestation, compared with those in the lowest quartile, BUN levels in the highest quartile decreased the risk of macrosomia and LGA by $60 \%(95 \% \mathrm{Cl}, 0.20-0.78), 66 \%(95 \% \mathrm{Cl}, 0.21,0.55)$, respectively, and increased the risk of SGA by $137 \%(1.06,5.31), 186 \%(1.29,6.34)$ in the third and fourth quartiles, respectively. The adjusted OR $(95 \% \mathrm{Cl})$ for macrosomia in the fourth quartile was $0.46(0.24,0.87)$, for SGA in the third quartiles was $2.36(1.10,5.10)$, and for LGA in the fourth quartile was $0.61(0.41,0.91)$ compared with those in the first quartile of SCr levels. The elevated changes of BUN $(>0.64 \mathrm{mmol} / \mathrm{L})$ was the risk factor of SGA (OR: 2.11, 95\%Cl: 1.03,4.32).

Conclusion: Higher BUN and SCr levels during the 28-30 th week of gestation even those towards the upper limit of the normal range can act as a warning sign of the impending SGA. Elevated changes of BUN and SCr during pregnancy also associated with the lower birth weight.

\section{Background}

In the process of pregnancy, the renal workload increases due to rise in maternal and fetus metabolites [1]. These physiological changes may lead to renal pathological damage and renal insufficiency, and the symptoms are relatively insidious [2-4]. A series of studies suggested that significant impairment of kidney function was independently associated with poor obstetric outcomes particularly preterm birth and growth restriction $[3,5-10]$. It has been recently reported that pregnancy women in the early stage of chronic kidney disease (CKD) orwith a mild decrease in glomerular filtration rate (GFR) were at an increased risk of adverse maternal and fetal events [5]. However, Kidney disease in pregnancy is often under-recognized and under-appreciated owing to the lack of symptoms and routine kidney function testing in pregnancy. Therefore, close monitoring of renal function during pregnancy plays an important role in reducing the risk of adverse maternal and infant events related to renal function.

BUN is the main end product of protein metabolism. The deamination of amino acids produces $\mathrm{NH}_{3}$ and $\mathrm{CO}_{2}$, which are synthesized into urea in the liver and then filter out of the glomeruli and excrete in the 
urine eventually [6]. BUN is used as a parameter to evaluate renal function since it will increase when renal efficiency is decompensated. A series of animal and epidemiological researches indicated that elevated BUN levels was a risk factor for insulin sensitivity reduction and increased the risk of diabetes and GDM [7-9]. However, the impact of BUN on fetal and maternal outcomes has not been established. $\mathrm{SCr}$, another indicator to assess renal function, is the product of muscle metabolism. In Muscle cells, creatine slowly forms $\mathrm{SCr}$ through irreversible non-enzymatic dehydration reaction, which is released into the blood and excreted by the kidney [6]. A recent large population-based cohort study in non-GDM pregnant women aged 18-45 years showed that higher SCr levels were related to decreased risk of macrosomia [10]. All told, there were limited data in the literature on the association between adverse pregnancy outcomes and maternal BUN and SCr levels. No research has paid attention to the influence of the change values during pregnancy and the combination of the two indicators on the pregnancy outcome as well.

In the present study, we aimed to examine the association of BUN and $\mathrm{SCr}$ in the second and third trimesters of pregnancy with adverse pregnancy outcomes including premature rupture of membranes (PROM), macrosomia, small for gestational age infants (SGA), large for gestational age infants (LGA), and to evaluate the influence of the combination of the two indicators and the changes between those two indicators in the second and third trimester of pregnancy on maternal and infant complications in a retrospective cohort study.

\section{Methods}

\section{Study design and participants}

A total of 1,701 pregnant women who registered and attended for their routine first hospital visit in pregnancy at Antenatal Department of Union Shenzhen Hospital of Huazhong University of Science and Technology (Shenzhen, Guangdong) and planned to give birth at this hospital were recruited from January 2015 to December 2018. The following exclusion criteria were included: history of liver disease $(n=45)$, diabetes or hypertension $(n=13)$, kidney disease $(n=5)$, heart disease $(n=5)$, twin or multiple pregnancy $(n=27)$. At last, a total of 1,606 gravidas with singleton pregnancies were included in the present study. The basic information of the participants was collected at the beginning, and serum BUN and $\mathrm{SCr}$ were measured in the second $\left(16-18^{\text {th }}\right)$ and third $\left(28-30^{\text {th }}\right)$ trimester of gestation respectively. And follow them up until delivery. Participants signed the informed consent at the beginning of the study with all procedures have been approved by the Ethics Committee of the Union Shenzhen Hospital of Huazhong University of Science and Technology.

\section{Collection of baseline information}

Age (years), education (primary, secondary, college or above), smoking status (yes or no), alcohol status (yes or no), conception method (natural or artificial), parity (primiparity or multiparity), history of miscarriage (yes or no), embryo number and history of disease (e.g., liver disease, diabetes or 
hypertension, kidney disease, heart disease) were obtained through face-to-face interviews by a welltraining investigator and questionnaires were completed simultaneously. Height and weight were measured using a electronic scale with detailed instructions to follow: stands up straight after taking off shoes with shoulder when she taking off shoes, stands up straight, shoulders parallel and the body is naturally relaxed and accurate to $0.1 \mathrm{~cm}$ and $0.1 \mathrm{~kg}$ respectively. Pre-pregnancy body mass index, BMI $\left(\mathrm{kg} / \mathrm{m}^{2}\right)$ was calculated as weight $(\mathrm{kg})$ divided by the square of the height $\left(\mathrm{m}^{2}\right)$.

\section{Laboratory assays}

At $16-18^{\text {th }}$ weeks and $28-30^{\text {th }}$ weeks of pregnancy, fasting venous blood were collected by professionally trained investigator. The samples were centrifuged at 3,500 rpm for 5 minutes at $4^{\circ} \mathrm{C}$ within 2 hours of collection. The BUN and SCr level were measured by enzymatic assay. All laboratory measurements were performed using accelerator a3600 automatic analyzer (Abbott, Chicago, USA).

\section{Assessment of pregnancy outcomes}

The adverse pregnancy outcomes of this study include: PROM, macrosomia, SGA and LGA. We followed the definition of the International Classification of Diseases, 10th Revision (ICD-10). The diagnostic criteria for PROM was that maternal self-report of vaginal fluid flow, vaginal PH $>6.5$ on litmus test paper, and amniotic fluid components on vaginal smear [11-13]. Macrosomia was diagnosed as a newborn with a birthweight greater than 4000g [14]. SGA and LGA were generally defined as below the $10^{\text {th }}$ or above the 90th percentile on the growth chart, respectively [15].

\section{Statistical analyses}

Baseline information was presented as means (SD) for continuous variables and proportion (\%) for categorical. Statistical difference between second trimester and third trimester were tested using Student's t-test. BUN and SCr were categorized by quartile distribution with the first quartile serving as the reference. Odds ratios (ORs) and $95 \%$ confidence intervals $(95 \% \mathrm{Cls}$ ) were calculated by using logistic regression models to examine the association of BUN and SCr of gestation with the risk of adverse pregnancy outcomes across each of the quartiles. Then, the differences (d-value) of the levels between BUN and SCr in the third and second trimester was calculated and analyzed in order to illustrate whether the risk of adverse pregnancy outcomes was caused by changing in concentration during pregnancy. Two models were included in the present study: Model 1 was unadjusted; Model 2 was adjusted for age, education, smoking status, alcohol status, conception method, parity and history of miscarriage. Restricted cubic spline(RCS) was used to reflect the nonlinear associations of BUN and SCr and d-value with adverse pregnancy outcomes. The data were regrouped based on the "cut-off value " of BUN and SCr in the RCS, Group1: no indicator exceeded the "cut-off value"; Group2: one of the indicators exceeded the "cut-off value"; Group3: indicators exceeded the "cut-off value", and the above two models were also constructed. Finally, a age-subgroup ( $<35$ and $\geq 35$ years) was performed because advanced maternal age is a known risk factor for adverse pregnancy outcomes [16]. All analyses were carried out by using SPSS 24.0 (SPSS Inc., Chicago, IL, USA) and a two-sided P-value $<0.05$ was considered statistically 
significant. Graphic production was completed by using R version 3.0.3 (The R Foundation for Statistical Computing, Vienna, Austria).

\section{Results}

\section{Baseline characteristics}

A total of 1606 singleton pregnant women aged 31.59 (3.83) years were included in the study with 308 cases of PROM, 95 cases of macrosomia, 65 cases of SGA, and 237 cases of LGA. As presented in Table 1, 383 cases were elderly pregnant women. The differences of BUN and SCr between the second trimester and third trimester were statistically significant $(P<0.05)$ and the levels of BUN and $\mathrm{SCr}$ in the third trimester were higher than those in the second trimester. 
Table 1

Baseline characteristics of all pregnant women in this study

\section{Characteristics of maternal and neonatal}

No. of maternal

Age(years)

Age categories

$<35$

$\geq 35$

Pre-pregnancy BMI $\left(\mathrm{kg} / \mathrm{m}^{2}\right)$

Education

Primary

Secondary

College or above

Smoking

Alcohol

Conception method

Natural

Artificial

Parity

Primiparity

Multiparity

History of miscarriage

BUN (mmol/L)

Second trimester

Third trimester

D-value
1606

31.59 (3.83)

1223 (76.2)

383 (23.8)

$20.86(3.34)$

$52(3.2)$

226 (14.1)

1328 (82.7)

$1(0.1)$

$3(0.2)$

1590 (99.0)

$12(0.7)$

711 (44.3)

867 (55.7)

$697(43.4)$

$2.61(0.62)^{a}$

$2.95(0.74)^{a}$

$0.34(0.75)$

Data was presented as mean(SD) for continuous variables and $n(\%)$ for categorical. Statistical difference between second trimester and third trimester were tested using Student's t-test.

Abbreviations: BMI: body mass index; BUN: serum urea nitrogen; SCr: serum creatinine; PROM:

premature rupture of membranes; SGA: small for gestational age; LGA: large for gestational age. ${ }^{a} p<$ 0.05 . 


\begin{tabular}{|c|c|}
\hline \multicolumn{2}{|c|}{ Characteristics of maternal and neonatal } \\
\hline \multicolumn{2}{|l|}{$\operatorname{SCr}(\mu \mathrm{mol} / \mathrm{L})$} \\
\hline Second trimester & $43.47(6.78)^{a}$ \\
\hline Third trimester & $47.54(7.98)^{a}$ \\
\hline D-value & $4.07(7.12)$ \\
\hline Birth weight (kg) & $3323.87(416.50)$ \\
\hline PROM & $308(19.2)$ \\
\hline Macrosomia & $95(5.9)$ \\
\hline SGA & $65(4.0)$ \\
\hline LGA & $237(14.8)$ \\
\hline \multicolumn{2}{|c|}{$\begin{array}{l}\text { Data was presented as mean(SD) for continuous variables and } n(\%) \text { for categorical. Statistical } \\
\text { difference between second trimester and third trimester were tested using Student's t-test. } \\
\text { Abbreviations: BMI: body mass index; BUN: serum urea nitrogen; SCr: serum creatinine; PROM: } \\
\text { premature rupture of membranes; SGA: small for gestational age; LGA: large for gestational age. a } p< \\
0.05 \text {. }\end{array}$} \\
\hline
\end{tabular}

\section{Association of BUN and SCr with adverse pregnancy outcomes}

The associations of BUN and $\mathrm{SCr}$ in the second trimester with adverse pregnancy outcomes were shown in Table 2 and Additional file Fig. 1. The second trimester BUN and SCr levels were not significant associated with PROM, macrosomia, SGA, or LGA. However, maternal with SCr levels in the fourth quartile had a $45 \%(95 \% \mathrm{Cl}, 1.01-2.09)$ higher risk of PROM than those in the first quartile. And each standard deviation (SD) of SCr levels increased the risk of PROM by $16 \%(95 \% \mathrm{Cl}, 1.02-1.32)$. Moreover, Additional file Fig. 1 indicated that the BUN level between 26.80 to $43.04 \mu \mathrm{mol} / \mathrm{L}$ was a protective factor to PROM. 
Table 2

ORs $(95 \% \mathrm{Cl})$ for the adverse pregnancy outcomes according to the quartiles of urea nitrogen (BUN), creatinine (SCr) in second trimester of pregnant

BUN (mmol/L)

$\begin{array}{llllll}\text { Q1 } & \text { Q2 } & \text { Q3 } & \text { Q4 } & P & \text { Per SD } \\ (<2.23) & (2.23-2.50) & (2.55-3.00) & (>3.00) & \text { trend } & \end{array}$

PROM

$\begin{array}{lllllll}\text { Case/N } & 85 / 459 & 76 / 350 & 82 / 449 & 65 / 348 & & \\ \text { Model } 1 & 1 & 1.22 & 0.98 & 1.01 & 0.796 & 0.95 \\ & \text { (reference) } & (0.86,1.73) & (0.70,1.38) & (0.71,1.45) & & (0.84,1.08) \\ \text { Model } 2 & 1 & 1.25 & 1.05 & 1.04 & 0.977 & 0.96 \\ & \text { (reference) } & (0.88,1.79) & (0.74,1.47) & (0.72,1.50) & & (0.85,1.10)\end{array}$

Macrosomia

$\begin{array}{lllllll}\text { Case/N } & 32 / 459 & 23 / 350 & 22 / 449 & 18 / 348 & & \\ \text { Model } 1 & 1 & 0.94 & 0.69 & 0.73 & 0.169 & 0.83 \\ & \text { (reference) } & (0.54,1.64) & (0.39,1.20) & (0.40,1.32) & & (0.67,1.04) \\ \text { Model 2 } & 1 & 0.89 & 0.72 & 0.69 & 0.163 & 0.82 \\ & \text { (reference) } & (0.50,1.56) & (0.41,1.26) & (0.38,1.27) & & (0.66,1.03)\end{array}$

SGA

$\begin{array}{lllllll}\text { Case/N } & 16 / 459 & 11 / 350 & 19 / 449 & 19 / 348 & & \\ \text { Model } 1 & 1 & 0.90 & 1.22 & 1.60 & 0.135 & 1.13 \\ & \text { (reference) } & (0.41,1.96) & (0.62,2.41) & (0.81,3.16) & & (0.89,1.43) \\ \text { Model } 2 & 1 & 0.93 & 1.17 & 1.53 & 0.207 & 1.10 \\ & \text { (reference) } & (0.42,2.04) & (0.59,2.33) & (0.76,3.08) & & (0.86,1.40)\end{array}$

LGA

\begin{tabular}{|c|c|c|c|c|c|c|}
\hline Case/N & $77 / 459$ & $57 / 350$ & $57 / 449$ & $46 / 348$ & & \\
\hline Model 1 & $\begin{array}{l}1 \\
\text { (reference) }\end{array}$ & $\begin{array}{l}0.97 \\
(0.66,1.40)\end{array}$ & $\begin{array}{l}0.72 \\
(0.50,1.05)\end{array}$ & $\begin{array}{l}0.76 \\
(0.51,1.22)\end{array}$ & 0.066 & $\begin{array}{l}0.88 \\
(0.77,1.02)\end{array}$ \\
\hline Model 2 & $\begin{array}{l}1 \\
\text { (reference) }\end{array}$ & $\begin{array}{l}0.89 \\
(0.61,1.32)\end{array}$ & $\begin{array}{l}0.73 \\
(0.50,1.06)\end{array}$ & $\begin{array}{l}0.75 \\
(0.50,1.21)\end{array}$ & 0.082 & $\begin{array}{l}0.88 \\
(0.76,1.02)\end{array}$ \\
\hline
\end{tabular}

Abbreviations: PROM: premature rupture of membranes; SGA: small for gestational age; LGA: large for gestational age. Model 1: without adjustment. Model 2: adjustment for age, pre-pregnancy BMI, education, smoking status, alcohol status, conception method, parity and history of miscarriage. 


\section{BUN (mmol/L)}

$\begin{array}{llll}\text { Q1 } & \text { Q2 } & \text { Q3 } & \text { Q4 } \\ (<38.80) & (38.80- & (43.10- & (>47.70) \\ & 43.00) & 47.70) & \end{array}$

PROM

$\begin{array}{lllllll}\text { Case/N } & 65 / 403 & 68 / 402 & 87 / 406 & 88 / 395 & & \\ \text { Model } 1 & 1 & 1.06 & 1.42 & 1.49 & 0.009 & 1.16 \\ & \text { (reference) } & (0.73,1.54) & (0.99,2.02) & (1.04,2.13) & & (1.03,1.32) \\ \text { Model } 2 & 1 & 1.06 & 1.39 & 1.45 & 0.019 & 1.16 \\ & \text { (reference) } & (0.73,1.56) & (0.96,2.00) & (1.01,2.09) & & (1.02,1.32)\end{array}$

Macrosomia

$\begin{array}{lllllll}\text { Case/N } & 25 / 403 & 25 / 402 & 25 / 406 & 20 / 395 & & \\ \text { Model } 1 & 1 & 1.01 & 0.99 & 0.81 & 0.513 & 0.91 \\ & \text { (reference) } & (0.57,1.78) & (0.56,1.76) & (0.44,1.48) & & (0.73,1.12) \\ \text { Model } 2 & 1 & 0.91 & 0.98 & 0.78 & 0.505 & 0.90 \\ & \text { (reference) } & (0.50,1.64) & (0.55,1.74) & (0.43,1.44) & & (0.73,1.12)\end{array}$

SGA

$\begin{array}{lllllll}\text { Case/N } & 13 / 403 & 21 / 402 & 9 / 406 & 22 / 395 & & \\ \text { Model } 1 & 1 & 1.65 & 0.68 & 1.77 & 0.370 & 1.14 \\ & \text { (reference) } & (0.82,3.35) & (0.29,1.61) & (0.88,3.56) & & (0.90,1.46) \\ \text { Model } 2 & 1 & 1.76 & 0.70 & 1.75 & 0.423 & 1.13 \\ & \text { (reference) } & (0.86,3.59) & (0.29,1.68) & (0.86,3.56) & & (0.88,1.45)\end{array}$

LGA

$\begin{array}{lllllll}\text { Case/N } & 62 / 403 & 70 / 402 & 53 / 406 & 52 / 395 & & \\ \text { Model } 1 & 1 & 1.16 & 0.83 & 0.83 & 0.165 & 0.91 \\ & \text { (reference) } & (0.80,1.69) & (0.56,1.23) & (0.56,1.24) & & (0.79,1.04) \\ \text { Model } 2 & 1 & 1.08 & 0.81 & 0.85 & 0.238 & 0.91 \\ & \text { (reference) } & (0.73,1.59) & (0.54,1.21) & (0.57,1.28) & & (0.79,1.05)\end{array}$

Abbreviations: PROM: premature rupture of membranes; SGA: small for gestational age; LGA: large for gestational age. Model 1: without adjustment. Model 2: adjustment for age, pre-pregnancy BMI, education, smoking status, alcohol status, conception method, parity and history of miscarriage.

The associations of BUN and $\mathrm{SCr}$ in the third trimester with adverse pregnancy outcomes were shown in Table 3 and Additional file Fig. 2. BUN levels were associated with the risk of macrosomia, SGA and LGA. Compared with those in the first quartile of BUN levels, OR $95 \% \mathrm{Cl}$ of macrosomia in the fourth quartile 
was $0.40(0.20-0.78)$, OR 95\% $\mathrm{Cl}$ of SGA in the third and fourth quartiles were 2.37 (1.06-5.31) and 2.86 (1.29-6.34), OR $95 \% \mathrm{Cl}$ of LGA in the highest quartile was $0.34(0.21,0.55)$. Each SD of BUN levels decreased the risk of macrosomia by $27 \%(95 \% \mathrm{Cl}, 0.57-0.92)$, increased the risk of SGA by $51 \%(95 \% \mathrm{Cl}$, $1.19-1.91)$, decreased the risk of LGA by $29 \%(95 \% \mathrm{Cl}, 0.61-0.83)$, respectively. And Additional file Fig. 2 showed that the BUN level between 2.89 to $5.20 \mathrm{mmol} / \mathrm{L}$ was a protective factor to macrosomia while between 2.91 to $5.20 \mathrm{mmol} / \mathrm{L}$ was a risk factor to $S G A$ in a non-linear manner. There was no statistically significant correlation between third trimester BUN and PROM. SCr levels were also associated with the risk of macrosomia, SGA and LGA. Compared with those in the first quartile of SCr levels, OR $95 \% \mathrm{Cl}$ of macrosomia in the fourth quartile was $0.46(0.24-0.87)$, OR 95\% $\mathrm{Cl}$ of SGA in the third quartiles was 2.36 (1.10-5.10), OR 95\% Cl of LGA in the fourth quartile was 0.61 (0.41-0.91). And each SD of SCr levels decreased the risk of macrosomia by $26 \%(95 \% \mathrm{Cl}, 0.59-0.93)$, increased the risk of SGA by $32 \%(95 \% \mathrm{Cl}$, $1.04-1.69)$, decreased the risk of LGA by $17 \%(95 \% \mathrm{Cl}, 0.71-0.96)$, respectively. And Additional file Fig. 2 showed that when SCr levels between 28.30 to $46.80 \mu \mathrm{mol} / \mathrm{L}$, it was a protective factor to SGA, but inversely related with LGA risk in a non-linear manner. Third trimester $\mathrm{SCr}$ levels was not statistically significant associated with risk for PROM. 
Table 3

ORs $(95 \% \mathrm{Cl})$ for the adverse pregnancy outcomes according to the quartiles of urea nitrogen (BUN), creatinine $(\mathrm{SCr})$ in third trimester of pregnant

\section{UN (mmol/L)}

$\begin{array}{llllll}\text { Q1 } & \text { Q2 } & \text { Q3 } & \text { Q4 } & P & \text { Per SD } \\ (<2.45) & (2.45-2.90) & (2.93-3.40) & (>3.40) & \text { trend } & \end{array}$

\section{PROM}

\begin{tabular}{|c|c|c|c|c|c|c|}
\hline Case/N & $85 / 406$ & $87 / 442$ & $60 / 392$ & $76 / 366$ & & \\
\hline Model 1 & $\begin{array}{l}1 \\
\text { (reference) }\end{array}$ & $\begin{array}{l}0.93 \\
(0.66,1.29)\end{array}$ & $\begin{array}{l}0.68 \\
(0.47,0.98)\end{array}$ & $\begin{array}{l}0.99 \\
(0.70,1.40)\end{array}$ & 0.537 & $\begin{array}{l}0.98 \\
(0.86,1.11)\end{array}$ \\
\hline Model & $\begin{array}{l}1 \\
\text { (reference) }\end{array}$ & $\begin{array}{l}0.89 \\
(0.63,1.26)\end{array}$ & $\begin{array}{l}0.65 \\
(0.45,0.95)\end{array}$ & $\begin{array}{l}0.88 \\
(0.61,1.26)\end{array}$ & 0.930 & $\begin{array}{l}0.94 \\
(0.83,1.07)\end{array}$ \\
\hline
\end{tabular}

Macrosomia

$\begin{array}{lllllll}\text { Case/N } & 33 / 406 & 29 / 442 & 20 / 392 & 13 / 366 & & \\ \text { Model } 1 & 1 & 0.79 & 0.61 & 0.42 & 0.005 & 0.75 \\ & \text { (reference) } & (0.47,1.33) & (0.34,1.08) & (0.22,0.80) & & (0.60,0.94) \\ \text { Model } 2 & 1 & 0.80 & 0.58 & 0.40 & 0.003 & 0.73 \\ & \text { (reference) } & (0.48,1.36) & (0.32,1.04) & (0.20,0.78) & & (0.57,0.92)\end{array}$

SGA

$\begin{array}{lllllll}\text { Case/N } & 9 / 406 & 14 / 442 & 20 / 392 & 22 / 366 & & \\ \text { Model } 1 & 1 & 1.44 & 2.37 & 2.82 & 0.003 & 1.43 \\ & \text { (reference) } & (0.62,3.37) & (1.07,5.27) & (1.28,6.21) & & (1.51,1.78) \\ \text { Model } 2 & \begin{array}{l}1 \\ \text { (reference) }\end{array} & \begin{array}{l}1.26 \\ (0.53,3.00)\end{array} & \begin{array}{l}2.37 \\ (1.06,5.31)\end{array} & \begin{array}{l}2.86 \\ (1.29,6.34)\end{array} & 0.002 & 1.51 \\ & & & & & (1.19,1.91)\end{array}$

LGA

\begin{tabular}{|c|c|c|c|c|c|c|}
\hline Case/N & $81 / 406$ & $66 / 442$ & $60 / 392$ & $30 / 366$ & & \\
\hline Model 1 & $\begin{array}{l}1 \\
\text { (reference) }\end{array}$ & $\begin{array}{l}0.70 \\
(0.49,1.01)\end{array}$ & $\begin{array}{l}0.73 \\
(0.50,1.05)\end{array}$ & $\begin{array}{l}0.36 \\
(0.23,0.56)\end{array}$ & 0.000 & $\begin{array}{l}0.72 \\
(0.62,0.84)\end{array}$ \\
\hline Model 2 & $\begin{array}{l}1 \\
\text { (reference) }\end{array}$ & $\begin{array}{l}0.78 \\
(0.54,1.21)\end{array}$ & $\begin{array}{l}0.79 \\
(0.54,1.15)\end{array}$ & $\begin{array}{l}0.34 \\
(0.21,0.55)\end{array}$ & 0.000 & $\begin{array}{l}0.71 \\
(0.61,0.83)\end{array}$ \\
\hline
\end{tabular}

Abbreviations: PROM: premature rupture of membranes; SGA: small for gestational age; LGA: large for gestational age. Model 1: without adjustment. Model 2: adjustment for age, pre-pregnancy BMI, education, smoking status, alcohol status, conception method, parity and history of miscarriage. 
$\mathrm{UN}(\mathrm{mmol} / \mathrm{L})$

$\begin{array}{llll}\text { Q1 } & \text { Q2 } & \text { Q3 } & \text { Q4 } \\ (<42.05) & (42.05- & (46.83- & (>52.25) \\ & 46.80) & 52.25) & \end{array}$

PROM

$\begin{array}{lllllll}\text { Case/N } & 68 / 406 & 73 / 397 & 85 / 402 & 82 / 401 & & \\ \text { Model } 1 & 1 & 1.12 & 1.33 & 1.28 & 0.114 & 1.08 \\ & \text { (reference) } & (0.78,1.61) & (0.94,1.90) & (0.90,1.82) & & (0.95,1.22) \\ \text { Model } 2 & 1 & 1.08 & 1.32 & 1.20 & 0.209 & 1.07 \\ & \text { (reference) } & (0.74,1.57) & (0.92,1.90) & (0.83,1.72) & & (0.94,1.22)\end{array}$

Macrosomia

\begin{tabular}{|c|c|c|c|}
\hline Case/N & $31 / 406$ & 23/397 & $25 / 402$ \\
\hline
\end{tabular}

$\begin{array}{lllllll}\text { Model } 1 & 1 & 0.74 & 0.80 & 0.50 & 0.045 & 0.77 \\ & \text { (reference) } & (0.43,1.30) & (0.47,1.39) & (0.27,0.93) & & (0.62,0.96)\end{array}$

$\begin{array}{lllllll}\text { Model } 2 & 1 & 0.75 & 0.77 & 0.46 & 0.024 & 0.74 \\ & \text { (reference) } & (0.43,1.31) & (0.44,1.34) & (0.24,0.87) & & (0.59,0.93)\end{array}$

SGA

$\begin{array}{lllllll}\text { Case/N } & 10 / 406 & 13 / 397 & 22 / 402 & 20 / 401 & & \\ \text { Model } 1 & 1 & 1.34 & 2.29 & 2.08 & 0.027 & 1.29 \\ & \text { (reference) } & (0.58,3.09) & (1.07,4.91) & (0.96,4.50) & & (1.04,1.62) \\ \text { Model 2 } & 1 & 1.34 & 2.36 & 1.97 & 0.040 & 1.32 \\ & \text { (reference) } & (0.58,3.12) & (1.10,5.10) & (0.90,4.32) & & (1.04,1.69)\end{array}$

LGA

\begin{tabular}{|c|c|c|c|c|c|c|}
\hline Case/N & $78 / 406$ & $54 / 397$ & $55 / 402$ & $50 / 401$ & & \\
\hline Model 1 & $\begin{array}{l}1 \\
\text { (reference) }\end{array}$ & $\begin{array}{l}0.66 \\
(0.45,0.97)\end{array}$ & $\begin{array}{l}0.67 \\
(0.46,0.97)\end{array}$ & $\begin{array}{l}0.60 \\
(0.41,0.88)\end{array}$ & 0.011 & $\begin{array}{l}0.82 \\
(0.72,0.96)\end{array}$ \\
\hline Model 2 & $\begin{array}{l}1 \\
\text { (reference) }\end{array}$ & $\begin{array}{l}0.75 \\
(0.51,1.10)\end{array}$ & $\begin{array}{l}0.71 \\
(0.48,1.05)\end{array}$ & $\begin{array}{l}0.61 \\
(0.41,0.91)\end{array}$ & 0.016 & $\begin{array}{l}0.83 \\
(0.71,0.96)\end{array}$ \\
\hline
\end{tabular}

Abbreviations: PROM: premature rupture of membranes; SGA: small for gestational age; LGA: large for gestational age. Model 1: without adjustment. Model 2: adjustment for age, pre-pregnancy BMI, education, smoking status, alcohol status, conception method, parity and history of miscarriage. 
The associations of changes for d-value of BUN and SCr with adverse pregnancy outcomes were shown in Table 4 and Additional file Fig. 3. D-value of BUN levels were associated with the risk of SGA and LGA. Compared with those in the first quartile of d-value for BUN levels, OR 95\% Cl of SGA in the fourth quartile was 2.11 (1.03-4.32), OR 95\% Cl of LGA in the fourth quartile was $0.58(0.34-0.87)$. And each SD of dvalue for BUN levels decreased the risk of SGA by $41 \%(95 \% \mathrm{Cl}, 1.01-1.80)$, decreased the risk of LGA by $29 \%(95 \% \mathrm{Cl}, 0.70-0.94)$ respectively. And Additional file Fig. 2 showed that the d-value of BUN Level from 0.31 to $2.45 \mathrm{mmol} / \mathrm{L}$ was a protective factor for SGA risk, while from 0.29 to $2.45 \mathrm{mmol} / \mathrm{L}$ was positively associated with risk of LGA in a non-linear manner. The associations of d-value of BUN in the third trimester with the risk of PROM and macrosomia was not statistically significant. 
Table 4

ORs $(95 \% \mathrm{Cl})$ for the adverse pregnancy outcomes according to the quartiles of the $\mathrm{d}$-value between the second and the third trimester

\begin{tabular}{|c|c|c|c|c|c|c|}
\hline & \multicolumn{4}{|c|}{ D-value of BUN(mmol/L) } & \multirow{3}{*}{$\begin{array}{l}P \\
\text { trend }\end{array}$} & \multirow[t]{3}{*}{ Per SD } \\
\hline & Q1 & Q2 & Q3 & Q4 & & \\
\hline & $(<-0.20)$ & $(-0.21-0.20)$ & $(0.21-0.63)$ & $(>0.64)$ & & \\
\hline \multicolumn{7}{|l|}{ PROM } \\
\hline Case/N & $67 / 374$ & $68 / 259$ & $73 / 394$ & $100 / 511$ & & \\
\hline Model 1 & $\begin{array}{l}1 \\
\text { (reference) }\end{array}$ & $\begin{array}{l}1.20 \\
(0.83,1.75)\end{array}$ & $\begin{array}{l}1.04 \\
(0.72,1.50)\end{array}$ & $\begin{array}{l}1.12 \\
(0.79,1.57)\end{array}$ & 0.735 & $\begin{array}{l}1.02 \\
(0.90,1.15)\end{array}$ \\
\hline Model 2 & $\begin{array}{l}1 \\
\text { (reference) }\end{array}$ & $\begin{array}{l}0.99 \\
(0.69,1.46)\end{array}$ & $\begin{array}{l}1.78 \\
(0.81,1.70)\end{array}$ & $\begin{array}{l}1.42 \\
(0.99,2.04)\end{array}$ & 0.033 & $\begin{array}{l}0.97 \\
(0.85,1.11)\end{array}$ \\
\hline \multicolumn{7}{|c|}{ Macrosomia } \\
\hline Case/N & $22 / 374$ & $22 / 327$ & $28 / 394$ & $23 / 511$ & & \\
\hline Model 1 & $\begin{array}{l}1 \\
\text { (reference) }\end{array}$ & $\begin{array}{l}1.15 \\
(0.63,2.13)\end{array}$ & $\begin{array}{l}1.22 \\
(0.69,2.18)\end{array}$ & $\begin{array}{l}0.75 \\
(0.41,1.38)\end{array}$ & 0.377 & $\begin{array}{l}0.89 \\
(0.72,1.10)\end{array}$ \\
\hline Model 2 & $\begin{array}{l}1 \\
\text { (reference) }\end{array}$ & $\begin{array}{l}1.13 \\
(0.61,2.10)\end{array}$ & $\begin{array}{l}1.18 \\
(0.66,2.11)\end{array}$ & $\begin{array}{l}0.68 \\
(0.37,1.27)\end{array}$ & 0.243 & $\begin{array}{l}0.87 \\
(0.70,1.09)\end{array}$ \\
\hline \multicolumn{7}{|l|}{ SGA } \\
\hline Case/N & $12 / 374$ & $9 / 327$ & $15 / 394$ & $29 / 511$ & & \\
\hline Model 1 & $\begin{array}{l}1 \\
\text { (reference) }\end{array}$ & $\begin{array}{l}0.85 \\
(0.36,2.05)\end{array}$ & $\begin{array}{l}1.19 \\
(0.55,2.59)\end{array}$ & $\begin{array}{l}1.82 \\
(0.91,3.61)\end{array}$ & 0.040 & $\begin{array}{l}1.33 \\
(1.05,1.67)\end{array}$ \\
\hline Model 2 & $\begin{array}{l}1 \\
\text { (reference) }\end{array}$ & $\begin{array}{l}0.92 \\
(0.38,2.28)\end{array}$ & $\begin{array}{l}1.56 \\
(0.70,3.49)\end{array}$ & $\begin{array}{l}2.11 \\
(1.03,4.32)\end{array}$ & 0.015 & $\begin{array}{l}1.41 \\
(1.10,1.80)\end{array}$ \\
\hline \multicolumn{7}{|l|}{ LGA } \\
\hline Case/N & $63 / 374$ & $50 / 327$ & $69 / 394$ & $55 / 511$ & & \\
\hline Model 1 & $\begin{array}{l}1 \\
\text { (reference) }\end{array}$ & $\begin{array}{l}0.89 \\
(0.59,1.34)\end{array}$ & $\begin{array}{l}1.05 \\
(0.72,1.53)\end{array}$ & $\begin{array}{l}0.60 \\
(0.40,0.88)\end{array}$ & 0.022 & $\begin{array}{l}0.82 \\
(0.71,0.94)\end{array}$ \\
\hline Model 2 & $\begin{array}{l}1 \\
\text { (reference) }\end{array}$ & $\begin{array}{l}0.91 \\
(0.60,1.39)\end{array}$ & $\begin{array}{l}1.10 \\
(0.74,1.62)\end{array}$ & $\begin{array}{l}0.58 \\
(0.34,0.87)\end{array}$ & 0.022 & $\begin{array}{l}0.81 \\
(0.70,0.94)\end{array}$ \\
\hline & \multicolumn{6}{|c|}{ D-value of $\mathrm{SCr}(\mu \mathrm{mol} / \mathrm{L})$} \\
\hline
\end{tabular}

Abbreviations: PROM: premature rupture of membranes; SGA: small for gestational age; LGA: large for gestational age. Model 1: without adjustment. Model 2: adjustment for age, pre-pregnancy BMI, education, smoking status, alcohol status, conception method, parity and history of miscarriage. 


\begin{tabular}{|c|c|c|c|c|c|c|}
\hline & \multicolumn{4}{|c|}{ D-value of BUN(mmol/L) } & \multirow{3}{*}{$\begin{array}{l}P \\
\text { trend }\end{array}$} & \multirow[t]{3}{*}{ Per SD } \\
\hline & Q1 & Q2 & Q3 & Q4 & & \\
\hline & $(<-0.20)$ & $(-0.21-0.20)$ & $(0.21-0.63)$ & $(>0.64)$ & & \\
\hline & Q1 & Q2 & Q3 & Q4 & & \\
\hline & $(<-0.5)$ & $(-0.48-3.70)$ & $(3.71-8.30)$ & $(>8.31)$ & & \\
\hline \multicolumn{7}{|l|}{ PROM } \\
\hline Case/N & $79 / 406$ & $81 / 399$ & 73/398 & $75 / 403$ & & \\
\hline Model 1 & $\begin{array}{l}1 \\
\text { (reference) }\end{array}$ & $\begin{array}{l}1.05 \\
(0.75,1.49)\end{array}$ & $\begin{array}{l}0.93 \\
(0.65,1.32)\end{array}$ & $\begin{array}{l}0.95 \\
(0.67,1.35)\end{array}$ & 0.609 & $\begin{array}{l}0.94 \\
(0.83,1.06)\end{array}$ \\
\hline Model 2 & $\begin{array}{l}1 \\
\text { (reference) }\end{array}$ & $\begin{array}{l}0.98 \\
(0.68,1.40)\end{array}$ & $\begin{array}{l}0.93 \\
(0.65,1.34)\end{array}$ & $\begin{array}{l}0.91 \\
(0.63,1.30)\end{array}$ & 0.557 & $\begin{array}{l}0.93 \\
(0.82,1.06)\end{array}$ \\
\hline \multicolumn{7}{|c|}{ Macrosomia } \\
\hline Case/N & $32 / 406$ & $25 / 399$ & $15 / 398$ & $23 / 403$ & & \\
\hline Model 1 & $\begin{array}{l}1 \\
\text { (reference) }\end{array}$ & $\begin{array}{l}0.78 \\
(0.45,1.34)\end{array}$ & $\begin{array}{l}0.46 \\
(0.24,0.86)\end{array}$ & $\begin{array}{l}0.71 \\
(0.41,1.23)\end{array}$ & 0.087 & $\begin{array}{l}0.83 \\
(0.67,1.03)\end{array}$ \\
\hline Model 2 & $\begin{array}{l}1 \\
\text { (reference) }\end{array}$ & $\begin{array}{l}0.82 \\
(0.47,1.41)\end{array}$ & $\begin{array}{l}0.43 \\
(0.22,0.82)\end{array}$ & $\begin{array}{l}0.18 \\
(0.39,1.20)\end{array}$ & 0.056 & $\begin{array}{l}0.81 \\
(0.66,1.01)\end{array}$ \\
\hline \multicolumn{7}{|l|}{ SGA } \\
\hline Case/N & $12 / 406$ & $13 / 399$ & $21 / 398$ & $19 / 403$ & & \\
\hline Model 1 & $\begin{array}{l}1 \\
\text { (reference) }\end{array}$ & $\begin{array}{l}1.11 \\
(0.50,2.45)\end{array}$ & $\begin{array}{l}1.83 \\
(0.89,3.77)\end{array}$ & $\begin{array}{l}1.63 \\
(0.78,3.39)\end{array}$ & 0.098 & $\begin{array}{l}1.20 \\
(0.95,1.53)\end{array}$ \\
\hline Model 2 & $\begin{array}{l}1 \\
\text { (reference) }\end{array}$ & $\begin{array}{l}1.04 \\
(0.46,2.36)\end{array}$ & $\begin{array}{l}1.85 \\
(0.89,3.84)\end{array}$ & $\begin{array}{l}1.62 \\
(0.77,3.41)\end{array}$ & 0.094 & $\begin{array}{l}1.23 \\
(0.96,1.58)\end{array}$ \\
\hline \multicolumn{7}{|l|}{ LGA } \\
\hline Case/N & $75 / 406$ & $59 / 399$ & $40 / 398$ & $63 / 403$ & & \\
\hline Model 1 & $\begin{array}{l}1 \\
\text { (reference) }\end{array}$ & $\begin{array}{l}0.76 \\
(0.53,1.11)\end{array}$ & $\begin{array}{l}0.49 \\
(0.33,0.74)\end{array}$ & $\begin{array}{l}0.82 \\
(0.57,1.18)\end{array}$ & 0.094 & $\begin{array}{l}0.90 \\
(0.78,1.04)\end{array}$ \\
\hline Model 2 & $\begin{array}{l}1 \\
\text { (reference) }\end{array}$ & $\begin{array}{l}0.83 \\
(0.56,1.21)\end{array}$ & $\begin{array}{l}0.49 \\
(0.32,0.74)\end{array}$ & $\begin{array}{l}0.82 \\
(0.56,1.20)\end{array}$ & 0.085 & $\begin{array}{l}0.89 \\
(0.77,1.03)\end{array}$ \\
\hline $\begin{array}{l}\text { Abbrevia } \\
\text { gestation } \\
\text { educatio }\end{array}$ & $\begin{array}{l}\text { ROM: pren } \\
\text { Model } 1: \mathrm{v} \\
\text { ing status, }\end{array}$ & $\begin{array}{l}\text { re rupture } \\
\text { ut adjust } \\
\text { hol statu }\end{array}$ & $\begin{array}{l}\text { hbranes; } \\
\text { lodel 2: a } \\
\text { eption me }\end{array}$ & $\begin{array}{l}\text { nall for ge } \\
\text { ent for ag } \\
\text { arity and }\end{array}$ & $\begin{array}{l}\text { onal ag } \\
\text { pregna } \\
y \text { of } m\end{array}$ & $\begin{array}{l}\text { LGA: large fo } \\
\text { cy BMI, } \\
\text { yarriage. }\end{array}$ \\
\hline
\end{tabular}


D-value of SCr levels were associated with the risk of macrosomia and LGA. Compared with those in the first quartile of d-value for SCr levels, OR $95 \% \mathrm{Cl}$ of macrosomia in the third quartile was $0.43(0.22-0.82)$, OR $95 \% \mathrm{Cl}$ of LGA in the third quartile was 0.49 (0.32-0.74). Additional file Fig. 3 showed that the d-value of $\mathrm{SCr}$ level from -13.40 to $3.65 \mathrm{mmol} / \mathrm{L}$ was inversely associated with the risk of both macrosomia and LGA in a non-linear manner. The associations of $d$-value of $\mathrm{SCr}$ in the third trimester with PROM and SGA were not statistically significant.

\section{Association of combined classification of BUN and SCr with adverse pregnancy outcomes}

The associations of combined classification of BUN and SCr with adverse pregnancy outcomes were shown in Table 5. The associations of combined classification of BUN and SCr in the third trimester with PROM were not statistically significant. However, compared with maternal in the $\mathrm{G} 1$, the ORs $95 \% \mathrm{Cl}$ of macrosomia in G2 and G3 were 0.59 (0.37-0.94), 0.46 (0.26-0.82), OR 95\% Cl of SGA in G3 were 3.34 (1.61-6.94), OR 95\% Cl of LGA in G2 and G3 were $0.71(0.51-0.98), 0.54(0.37-0.79)$. 
Table 5

ORs $(95 \% \mathrm{Cl})$ for the adverse pregnancy outcomes according to the group of the combination of urea nitrogen (BUN) and creatinine (SCr) in the third trimester

\section{Groups of BUN and SCr}

$P_{\text {trend }}$

G1

G2

G3

PROM

Case/N

$92 / 495$

$129 / 661$

$87 / 450$

Model 1

1 (reference)

$1.06(0.79,1.43)$

$1.05(0.76,1.45)$

0.764

Model 2

1 (reference)

$1.10(0.81,1.49)$

$0.98(0.70,1.37)$

0.913

Macrosomia

\begin{tabular}{lllll} 
Case $/ \mathrm{N}$ & $41 / 495$ & $34 / 661$ & $20 / 450$ & \\
Model 1 & 1 (reference) & $0.60(0.38,0.96)$ & $0.52(0.30,0.89)$ & 0.012 \\
\hline Model 2 & 1 (reference) & $0.59(0.37,0.94)$ & $0.46(0.26,0.82)$ & 0.005 \\
SGA & & & &
\end{tabular}

\section{Case/N}

10/495

$26 / 661$

$29 / 450$

Model 1

1 (reference)

$1.99(0.95,4.16)$

$3.34(1.61,6.94)$

0.001

Model 2

1 (reference)

$1.93(0.91,4.08)$

$3.49(1.67,7.31)$

0.001

LGA

$\begin{array}{lllll}\text { Case/N } & 93 / 495 & 93 / 661 & 51 / 450 & \\ \text { Model } 1 & 1 \text { (reference) } & 0.71(0.52,0.97) & 0.55(0.38,0.80) & 0.001 \\ \text { Model } 2 & 1 \text { (reference) } & 0.71(0.51,0.98) & 0.54(0.37,0.79) & 0.001\end{array}$

PROM: premature rupture of membranes; SGA: small for gestational age; LGA: large for gestational age. Model 1: without adjustment. Model 2: adjustment for age, pre-pregnancy BMI, education, smoking status, alcohol status, conception method, parity and history of miscarriage.

Subgroups younger than 35 years old had the same associations as above, but most subgroups older than 35 years old were not statistically significant (Additional file Table 1, 2). And statistical tests for interactions between BUN/SCr and age on outcome were not significant (all $P>0.05$ ).

\section{Discussion}

In this retrospective cohort study, we observed an increased risk of PROM in pregnant women with high or even those towards the upper limit of the normal range of SCr levels during 16-18th weeks of gestation. 
During 28-30th weeks of gestation, pregnant women with higher or even those towards the upper limit of the normal range of BUN and SCr exhibited an increased risk of SGA. The elevated changes of BUN during pregnancy influenced the birth weight of newborn. In addition, the risk of SGA increased substantially if BUN and SCr levels high or even those towards the upper limit of the normal range simultaneously.

Mild renal function damage would lead to improper regulation of gestational adaptation to volume and vascular pressure change, resulting in obstetric complications [17]. And as kidney function declines, the chance of a women having adverse maternal outcomes increased [18]. A retrospective study conducted by Jessica Kendrick et al. found that women with kidney disease increased the incidence of low birth weight by $138 \%$ (95\% Cl, 1.64-3.44), increased incidence of SGA by $37 \%(95 \% \mathrm{Cl}, 0.94-2.00)$ [19]. In addition, a recently systematic review and meta-analysis showed that pregnancy with CKD had greater odds of premature delivery (OR, 5.72; $95 \% \mathrm{Cl}, 3.26-10.03)$, small for gestational age/low birth weight (OR, $4.85 ; 95 \% \mathrm{Cl}, 3.03-7.76)[17]$.

At present, the reference for biochemical indexes of pregnant women was no unified.[20-22] A recent cross-sectional study of 13656 healthy pregnant women and 2634 non-pregnant women pointed out that the median of BUN at $28-35$ weeks of gestation was $2.7 \mathrm{mmol} / \mathrm{L}(95 \% \mathrm{Cl} 1.6-4.3)$, and the median of SCr at $13-20$ and $28-35$ weeks of gestation was $43.27 \mu \mathrm{mol} / \mathrm{L}(95 \% \mathrm{Cl} 31.3-57.6), 40.27 \mu \mathrm{mol} / \mathrm{L}(95 \% \mathrm{Cl}$ 30.3-53.1) [22]. It is worth noting that our study showed that the critical value of increased risk of PROM caused by SCr levels in the second trimester was $47.70 \mu \mathrm{mol} / \mathrm{L}$, while the critical value of increased risk of SGA caused by BUN and SCr levels in the third trimester was $2.9 \mathrm{mmol} / \mathrm{L}$ and $46.80 \mu \mathrm{mol} / \mathrm{L}$, respectively. Hence,higher BUN and SCr levels or even close to the upper limit of the normal range, could give an early warning of occurrence for SGA. Renal disease is often clinically silent in the early and middle stage of renal injury. Bun and SCr may change only slightly until GFR drops by more than $50 \%$. However, these pathological changes affect the regulation of blood pressure and blood volume, which can easily lead to insufficient uterine placental perfusion and affect fetal development. The exact mechanism associated with this issue was not well understood and requires further studies.

The increase of an index may be greatly influenced by external factors, thus we combine the BUN and SCr measurements and regroup them for analysis. The risk of SGA increased by $249 \%$ in pregnant women whose two indicators exceeded the cut-off value, compared with those whose two indicators did not exceed the cut-off value. We also analyzed the relative BUN and $\mathrm{SCr}$ change from the second trimester to the third trimester of gestation age. According to the Table 5 and Supplementary Fig. 3, the risk of SGA was higher with a elevated meteral BUN level of $>0.64 \mathrm{mmol} / \mathrm{L}$. Therefore, not only the real-time level of renal function indicators must be paid attention to, but also the dynamic monitoring of renal function. $A$ interaction and subgroup analysis by age ( $<35$ and $\geq 35$ years) was also performed to verify whether age would affect the risk of adverse pregnancy outcomes in association with maternal BUN and SCr levels. We found a significant correlation between BUN and SCr levels and adverse pregnancy outcomes in women aged $<35$ years, but not in women aged $\geq 35$ years. There was no significant interaction between maternal age and the level of BUN and SCr (all P-interaction >0.05). Further studies with larger sample 
sizes are required to clarify the risk of adverse pregnancy outcomes in relation to BUN and Scr levels and age dependent effects.

Although our study comprehensively explored the association between maternal renal function using two parameters and the risk of adverse pregnancy outcomes in a relatively large sample size, some limitations in this study remain. Firstly, the analytic cohort were from China, which may limit the generalizability of the study results. Secondly, although we accounted for known confounders, some unmeasured or unknown residual confounders remained (either unmeasured or unknown). Finally, the concentrations of BUN and SCr were greatly influenced by other factors, and did not change significantly when GFR was slightly decreased, so they were not sensitive indicators of renal damage. However,the chosen biochemical parameters of BUN and SCr to assess maternal renal function are simple, inexpensive and readily available tests thus should be additionally evaluated [23].

\section{Conclusions}

Higher BUN and SCr levels during the 28-30th week of gestation even those towards the upper limit of the normal range can act as a warning sign of the impending SGA. Elevated changes of BUN and SCr during pregnancy also associated with the lower birth weight. Combined analysis of the two indicators is more conducive to evaluate renal function and prevent the occurrence of SGA. More attention should be paid to the dynamic monitoring of renal function during pregnancy.

\section{Abbreviations}

BMI: body mass index; BUN: Blood urea nitrogen; CKD: Chronic kidney disease; GDM: Gestational diabetes mellitus; GFR: Glomerular filtration rate; LGA: Large for gestational age infants; SCr: Serum creatinine; SD: standard deviation; SGA: Small for gestational age infants; ORs: Odds ratios 95\%; Cls: 95\% confidence intervals; PROM: Premature rupture of membranes; RCS: Restricted cubic spline

\section{Declarations}

\section{Ethics approval and consent to participate}

The protocol for this study was approved by the Ethics Committee of the Union Shenzhen Hospital of Huazhong University of Science and Technology (No. 2019072644).

\section{Consent for publication}

Not applicable

\section{Availability of data and materials}

The datasets used and/or analyzed during the current study are available from the corresponding author on reasonable request. 


\section{Competing interests}

The authors declare that they have no competing interests

\section{Funding}

This work was supported by Shenzhen Nanshan District Science and Technology Project [grant number 2019007]. The funding sources had no involvement in the study design; collection, analysis, or interpretation of data; the writing of the report, nor the decision to submit the article for publication.

\section{Authors' contributions}

GD, ZL and LW conceived and designed the study. YW and RS assisted with study design. LW contributed to statistical analysis and wrote the manuscript. YL and $\mathrm{HC}$ provided statistical advice and assisted with data analysis. GD and SS reviewed and edited the manuscript. All authors read and approved the final manuscript.

\section{Acknowledgments}

We thank the mothers and children who participated in this study and all the clinical staff at Union Shenzhen Hospital of Huazhong University of Science and Technology for their support and contribution.

\section{References}

1. Kramer MS. The Epidemiology of Adverse Pregnancy Outcomes: An Overview. Journal of Nutrition 2003, 133(5 Suppl 2):1592S.

2. Ganchimeg T, Ota E, Morisaki N, Laopaiboon M, Lumbiganon P, Zhang J, Yamdamsuren B, Temmerman M, Say L, Tuncalp $O$ et al. Pregnancy and childbirth outcomes among adolescent mothers: a World Health Organization multicountry study. BJOG 2014, 121 Suppl 1:40-48.

3. Bramham K, Parnell B, Nelson-Piercy C, Seed PT, Poston L, Chappell LC. Chronic hypertension and pregnancy outcomes: systematic review and meta-analysis. BMJ 2014, 348:g2301.

4. Odutayo A, Hladunewich M. Obstetric nephrology: renal hemodynamic and metabolic physiology in normal pregnancy. Clin J Am Soc Nephrol 2012, 7(12):2073-2080.

5. Park S, Lee SM, Park JS, Hong JS, Chin HJ, Na KY, Kim DK, Oh KH, Joo KW, Kim YS et al. Gestational Estimated Glomerular Filtration Rate and Adverse Maternofetal Outcomes. Kidney Blood Press Res 2018, 43(5):1688-1698.

6. Webster AC, Nagler EV, Morton RL, Masson P. Chronic Kidney Disease. The Lancet 2017, 389(10075):1238-1252.

7. Xie Y, Bowe B, Li T, Xian H, Yan Y, Al-Aly Z. Higher blood urea nitrogen is associated with increased risk of incident diabetes mellitus. Kidney Int 2018, 93(3):741-752. 
8. Koppe L, Pelletier CC, Alix PM, Kalbacher E, Fouque D, Soulage CO, Guebre-Egziabher F. Insulin resistance in chronic kidney disease: new lessons from experimental models. Nephrol Dial Transplant 2014, 29(9):1666-1674.

9. Feng P, Wang G, Yu Q, Zhu W, Zhong C. First-trimester blood urea nitrogen and risk of gestational diabetes mellitus. J Cell Mol Med 2020, 24(4):2416-2422.

10. Hua XG, Jiang W, Hu R, Hu CY, Huang K, Li FL, Zhang XJ. Large for gestational age and macrosomia in pregnancies without gestational diabetes mellitus. J Matern Fetal Neonatal Med 2020, 33(21):3549-3558.

11. Goldenberg RL, Culhane JF, lams JD, Romero R. Epidemiology and causes of preterm birth. Lancet 2008, 371(9606):75-84.

12. Liu J, Feng ZC, Wu J. The incidence rate of premature rupture of membranes and its influence on fetal-neonatal health: a report from mainland China. J Trop Pediatr 2010, 56(1):36-42.

13. Zhou Q, Zhang W, Xu H, Liang H, Ruan Y, Zhou S, Li X. Risk factors for preterm premature rupture of membranes in Chinese women from urban cities. Int J Gynaecol Obstet 2014, 127(3):254-259.

14. Glodean DM, Miclea D, Popa AR. Macrosomia. A Systematic Review of Recent Literature. Romanian Journal of Diabetes Nutrition and Metabolic Diseases 2018, 25(2):187-195.

15. He JR, Xia HM, Liu Y, Xia XY, Mo WJ, Wang P, Cheng KK, Leung GM, Feng Q, Schooling CM et al. A new birthweight reference in Guangzhou, southern China, and its comparison with the global reference. Arch Dis Child 2014, 99(12):1091-1097.

16. Frederiksen LE, Ernst A, Brix N, Braskhoj Lauridsen LL, Roos L, Ramlau-Hansen CH, Ekelund CK. Risk of Adverse Pregnancy Outcomes at Advanced Maternal Age. Obstet Gynecol 2018, 131(3):457-463.

17. Zhang JJ, Ma XX, Hao L, Liu LJ, Lv JC, Zhang H. A Systematic Review and Meta-Analysis of Outcomes of Pregnancy in CKD and CKD Outcomes in Pregnancy. Clin J Am Soc Nephrol 2015, 10(11):1964-1978.

18. Piccoli GB, Attini R, Vasario E, Conijn A, Biolcati M, D'Amico F, Consiglio V, Bontempo S, Todros T. Pregnancy and chronic kidney disease: a challenge in all CKD stages. Clin J Am Soc Nephro/2010, 5(5):844-855.

19. Kendrick J, Sharma S, Holmen J, Palit S, Nuccio E, Chonchol M. Kidney disease and maternal and fetal outcomes in pregnancy. Am J Kidney Dis 2015, 66(1):55-59.

20. Jia L, Yongmei J, Leiwen $P, Y i$ Y. The reference intervals for renal function indexes in chinese pregnant women. Pakistan Journal of Pharmaceutical Sciences 2017, 30(3(Special)):1133.

21. Hayashi M, Ueda Y, Hoshimoto K, Ota Y, Fukasawa I, Sumori K, Kaneko I, Abe S, Uno M, Ohkura T et al. Changes in urinary excretion of six biochemical parameters in normotensive pregnancy and preeclampsia. Am J Kidney Dis 2002, 39(2):392-400.

22. Dai Y, Liu J, Yuan E, Li Y, Wang Q, Jia L, Wang L, Su Y. Gestational age-specific reference intervals for 15 biochemical measurands during normal pregnancy in China. Ann Clin Biochem 2018, 55(4):446452. 
23. Nagalakshmi CS, Devaki RN, Akila P, Suma KB, Sujatha P. Exploration of the Clinico-Biochemical Parameters to Explain the Altered Renal Mechanisms in Gestational Diabetes Mellitus. Journal of Clinical \& Diagnostic Research 2012, 6(3):369-371.

\section{Supplementary Files}

This is a list of supplementary files associated with this preprint. Click to download.

- Additionalfiles.docx 